Associated Universities, Inc.

Upton, New York 11973

RHIC TeChNical Note No. 41

\author{
Disassociation of Electrons from Partially \\ Stripped Ion Beams \\ Due to Strong Magnetic Fields
}

S. Tepikian and S. Y. Lee

July 1988 


\title{
DISASSOCIATION OF ELECTRONS FROM PARTIALLY \\ STRIPPED ION BEAMS \\ DUE TO STRONG MAGNETIC FIELDS
}

\author{
S. Tepikian and S. Y. Lee
}

Brookhaven National Laboratory

Upton, New York 11973

\begin{abstract}
We study the probability of disassociating an electron from partially stripped ions in the AGS. Our calculation indicates that the probability for electron disassociation due to the strong external magnetic field in the AGS is very small for tightly bounded electrons. Two methods, a one-dimensional JWKB method and an energy density approximation, are used estimate this effect. Both methods yield consistant picture to support the idea of accelerating the partially stripped ions in the AGS, which requires some upgrade in the vacuum pressure due to other processes such as electron capture and ionization.
\end{abstract}




\section{Introduction}

Recently $y^{1}$, a new scenario for the heavy ion operation in the Booster AGS - RHIC has been proposed, where partially stripped ions will be accelerated in the AGS. The advantage of this scenario is that the heavy ion operation in the RHIC would be less source limited in the Tandem and the space charge limit in the Booster is also increased. The scenario can also eliminate the need of $h=1 \mathrm{r} . \mathrm{f}$. system in the Booster. The disadvantage of this scenario is that the partially stripped ion has to be accelerated in the AGS.

Acceleration of partially stripped ions would encounter several problems. Rhoades-Brown and Gould ${ }^{1}$ has analyzed the effect of the electron capture and electron stripping due to the vacuum pressure of the AGS. It is known also that when partially stripped ions are moving in strong electromagnetic fields, the atomic structure is distorted and an electron may be removed.

This paper shall study the problem of electron stripping by an external magnetic field from two different points of view: (1) penetration of a potential barrier (section 2) and (2) the energy density of the ion and external fields (section 3). In section 4, we estimate the life time for an electron. The conclusion will be given in section 5 .

\section{Barrier Penetration}

The partially stripped heavy ion beam (where each ion is assumed to have only two electrons) in an accelerator, moving with the velocity $c \beta$ along the beam pipe, experiences a transverse magnetic guide field. See Fig. 1. Both electrons are bound to the nucleus. The binding energy for a single electron is given below 2

$$
E_{B I N D}=\mu c^{2}\left[\left[1+\frac{\alpha^{2}\left(Z-\frac{5}{16}\right)^{2}}{\left[s+\sqrt{\left(\ell+\frac{1}{2}\right)^{2}-\alpha^{2}\left(Z-\frac{5}{16}\right)^{2}}+\frac{1}{2}\right]^{2}}\right]^{-1 / 2}-1\right]
$$

where $Z$, the charge number of the nucleus, is effectively reduced by $5 / 16$ to take into account the electron-electron repulsive force ${ }^{3} \mu$ is the reduced mass, $c$ is the speed of light, $\alpha$ is the fine structure constant and $s$ and $\ell$ are spin and orbital angular momentum quantum numbers respectively. Figure 2 shows a plot of $E_{B I N D}$ versus $Z$ for ground state electrons.

The $E_{\text {BIND }}$ is defined in the center of mass frame of the ion. For consistancy we transform the magnetic guide field of the accelerator to the center of mass frame using ${ }^{4}$ 


$$
\begin{aligned}
& E_{C M}=\gamma(E+\beta \times B)-\frac{\gamma^{2}}{\gamma+1} \beta(\beta \cdot E) \\
& B_{C M}=\gamma(B-\beta \times E)-\frac{\gamma^{2}}{\gamma+1} \beta(\beta \cdot B)
\end{aligned}
$$

where $\gamma$ is the lorentz factor, $\beta$ is the velocity divided by $c, B$ and $E$ are the fields in the laboratory frame and $B_{C M}$ and $E_{C M}$ are the fields in the center of mass frame of the ion. Since $E=0$ and $B=-B_{z} z$ then

$$
\begin{aligned}
& E_{C Y}=-\gamma \beta B_{z} \hat{x} \\
& B_{C M}=-\gamma \beta B_{z} \hat{z}
\end{aligned}
$$

where $\hat{\mathbf{x}}, \hat{\mathbf{s}}$ and $\hat{\mathbf{z}}$ are mutually perpendicular unit vectors in the local coordinate system and the ion is moving in the $\hat{\mathbf{s}}$ direction.

The force acting on an electron due to the external fields is

$$
F_{e}=e\left(E_{C M}+\beta_{e} \times B_{C H}\right)
$$

where $\beta_{e}$ is the electron speed in the center of mass frame. The magnetic field will induce the strong Zeeman effect of eliminating the magnetic splitting, while the electric field would induce the Stark effect, atomic polarization and even electron stripping. At relativistic energy, these two effects add up coherently. In the center of mass frame the work done on an electron due to the external fields is

$$
W=\operatorname{er} \beta(1+\beta) B_{z} x
$$

where $x$ is the coordinate along the $\hat{\mathbf{x}}$ direction. Using $W$ we find an electron on the $x$ axis experiences the following potential energy

$$
U_{e}=\operatorname{er\beta }(1+\beta) B_{z} x-\frac{e^{2} z}{|x|}-E_{B I N D} .
$$


When $U>0$ the the electron can be unstable and stripped from the ion. Figure 3 shows a graph of $U_{e}$ versus $x$ where we notice that there is a barrier in region II. If an electron can penetrate this barrier then it can be stripped from the ion. We estimate transmission through the barrier with ${ }^{5}$

$$
T=\exp \left\{-\frac{4 \pi}{h} \int_{x_{1}}^{x_{2}} \sqrt{2 \mu U_{e}(x)} d x\right\}
$$

where $x_{1}$ and $x_{2}$ are the boundaries of the barrier, $\mu$ is the mass of an electron and $h$ is Planck's constant. The expressions for $x_{1}$ and $x_{2}$ are given below (i.e. when $U_{e}(x)=0$ )

$$
\begin{aligned}
& x_{1}=\frac{E_{\text {BIND }}-\sqrt{E_{\text {BIND }}^{2}-4 e^{3} \gamma \beta(1+\beta) B_{Z} Z}}{2 e \gamma \beta B_{z}} \\
& x_{2}=\frac{E_{B I N D}+\sqrt{E_{B I N D}^{2}-4 e^{3} \gamma \beta(1+\beta) B_{z} Z}}{2 e \gamma \beta B_{Z}} .
\end{aligned}
$$

The transmission $\mathrm{T}$ gives us an estimate for electron disassociation from the ions. We look at various ion species in the AGS at various energy ranges to find the probability of an ion being stripped. The results are given by the solid lines in Figs. 4-5. Only in very extreme cases do we find any possibility of stripping. In general the effect is not important.

\section{Energy Density}

Alternatively, the ion can be modeled in a stable state within a volume V. The energy density felt by a both electrons due to the nucleus can be expressed as

$$
U_{\text {ion }}=2 \frac{E_{B I N D}}{V}
$$

where 2 represents two electrons in the partially stripped ion. The energy density is negative, so that electrons are bounded. When the external field is applied to the partially stripped ion, the energy density may change sign causing the electron to become unstable. The energy density due to the external fields which can be expressed in the center of mass frame as 


$$
U_{\text {fields }}=\frac{1}{8 \pi}\left(\left|E_{C M}\right|^{2}+\left|B_{C M}\right|^{2}\right)=\frac{1}{8 \pi} \gamma^{2} B_{z}^{2}\left(1+\beta^{2}\right)
$$

The total energy density becomes

$$
U=U_{f i e l d s}+U_{i \text { in }}=\frac{1}{8 \pi} \gamma^{2} B_{z}^{2}\left(1+\beta^{2}\right)+2 \frac{E_{B I N D}}{V} .
$$

The electron is stable as long as $U<0$ otherwise the electron may be stripped. We can define a critical volume such that $U=0$ as

$$
V_{c}=-\frac{16 \pi E_{B I N D}}{\gamma^{2} B_{z}^{2}\left(1+\beta^{2}\right)}
$$

An electron found outside this volume is expected to be stripped. If this volume is assumed to be a sphere then the electron must reside within the following radius

$$
r_{C}=\left[-\frac{12 E_{B I N D}}{\gamma^{2} B_{z}^{2}\left(1+\beta^{2}\right)}\right]^{1 / 3}
$$

for it to remain bounded.

We now find the probability of an electron to be outside this volume. This should reflect the probability that the electron can be stripped. Given the wave function, $\psi$, of the electron then

$$
P=\int_{r>r_{c}} \psi^{*} \psi d \tau
$$

The propability $P$ can be estimated from the ground state wave function for the Hydrogen atom with a nucleus of $\mathrm{Z}$ protons. This gives:

$$
P=\left[1+2 \frac{r_{c}\left(z-\frac{5}{16}\right)}{a_{0}}+2\left[\frac{r_{c}\left(z-\frac{5}{16}\right)}{a_{0}}\right]^{2}\right] e^{-2\left(z-\frac{5}{16}\right) r_{c} / a_{0}}
$$

where $a_{0}$ is the Bohr radius of the Hydrogen atom. 
The results from this probability are also shown in Figs. 4-5 along with the transmission derived in sect 2. The probability found by this method is exceedingly small and thus shows no effect. In comparing the two methods, the results disagree in quantitatively but reach the same conclusion that the probability of electron disassociation is exceedingly small in the AGS. The differences between the methods can come in because of the different assumptions used in both models, such as, the choice of the shape of the volume and some non-relativistic approximations. For example, if we use the non-relativistic binding energy, in the barrier penetration calculation of section 3 , the penetrability integral will reduced by a factor of 2 .

\section{Life-time Estimation}

To calculate the life time, one should solve the eigenvalue of the electron in the complex energy formulation. Since the probability is relatively small, the stripping rate, $\lambda$, can be approximated by

$$
\lambda=f P
$$

where $f$ is the frequency of the electron oscillation and $P$ is the penetrability discussed in sections 2 and 3 .

To estimate $f$, we use the result that the kinetic energy is equal to half of the potential energy,

$$
v=\frac{e Z_{\text {eff }}}{\sqrt{\mu a_{0}}}
$$

Whence, the time required for the electron to traverse a distance of the Bohr radius for $\mathrm{K}$ shell electron is

$$
\tau=\frac{4 a_{0} / Z_{\text {eff }}}{v}=\frac{4 a_{0} \sqrt{\mu a_{0}}}{e Z_{\text {eff }}^{2}}
$$

The frequency then becomes

$$
f=\frac{e z_{e f f}^{2}}{4 a_{0} \sqrt{\mu a_{0}}}=1.0 \times 10^{16} z_{e f f}^{2} \quad\left(\mathrm{sec}^{-1}\right)
$$

Multipying $f$ and $P$ (see Fig. 4) together for $Z=79$, we obtain the electron disassociation rate to be effectively zero. The effect can be important only when the energy and magnetic fields are large, such as $\gamma B \geq 10^{7}$ Tesla (see Fig. 5), where the stripping rate may become sizable. Some ions generated in the supernova explosion may encounter conditions where the stripping is important in the astronomical events 


\section{Conclusion}

We have studied the possibility of electron disassociation by the dipole guide field acting on partially stripped ion beams. We found that the electron disassociation probability is very small for ions with tightly bound electrons. The electrons bounded in the $\mathrm{K}$ shell, experiences an electric field is $2.7 \mathrm{z}_{\text {eff }}^{3} \times 10^{11} \mathrm{~V} / \mathrm{m}$. The corresponding strength the static magnetic field is $3 \times 10^{8} \mathrm{rB}_{\mathrm{z}}[\mathrm{T}] \mathrm{V} / \mathrm{m}$. Thus the effect of the electron disassociation is small except for loosely bounded electrons, such as $\mathrm{H}^{-}$, or extraodinarly large $\gamma \mathrm{B}_{z}$.

\section{References}

1. M. Rhoades-Brown and H. Gould, AD/RHIC-38

2. Condon and G. H. Shortly, The Theory of Atomic Spectra, 4'th ed. (Cambridge University Press, 1957)

3. A. Messiah, Quantum Mechanics, (John Wiley \& Sons, Inc., 1958)

4. J. D. Jackson, Classical Electrodynamics, 2nd ed. (John Wiley \& Sons, Inc., 1975)

5. R. M. Eisberg, Fundumentals of Modern Physics, (John Wiley \& Sons, Inc., 1961) 


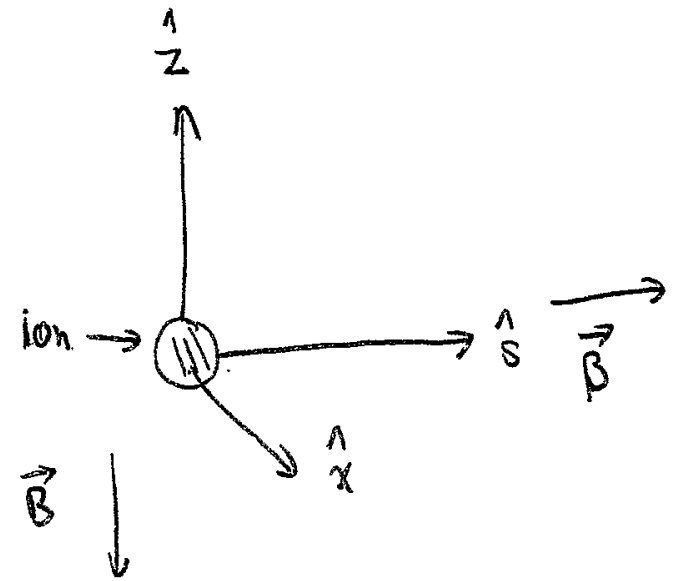

Figure 1 The coordinate system 


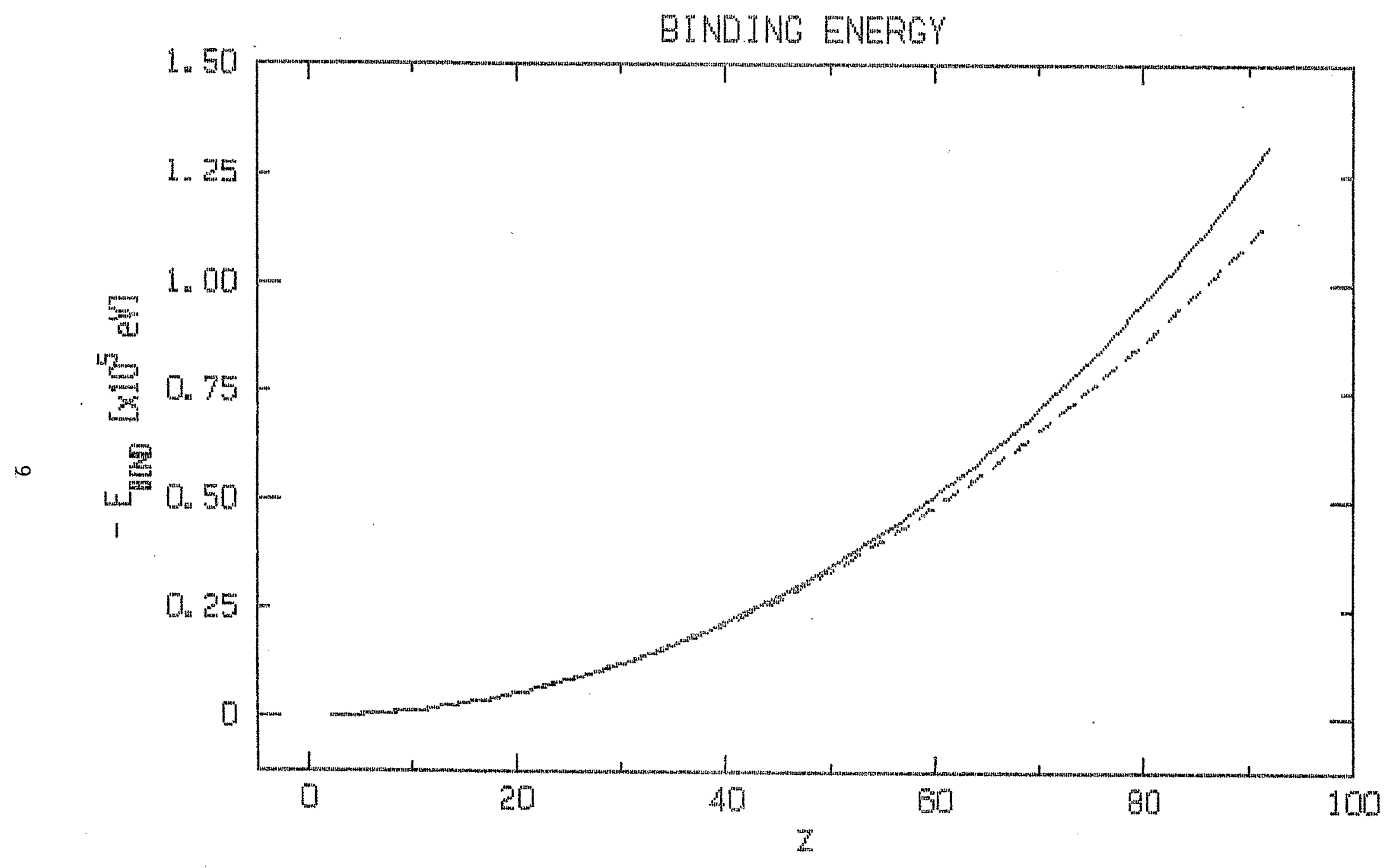

Figure 2 The binding energy versus the nuclear charge. The solid line is the electron binding energy from the Dirac equation. The dashed line is the binding energy from the Schrödinger equation. 


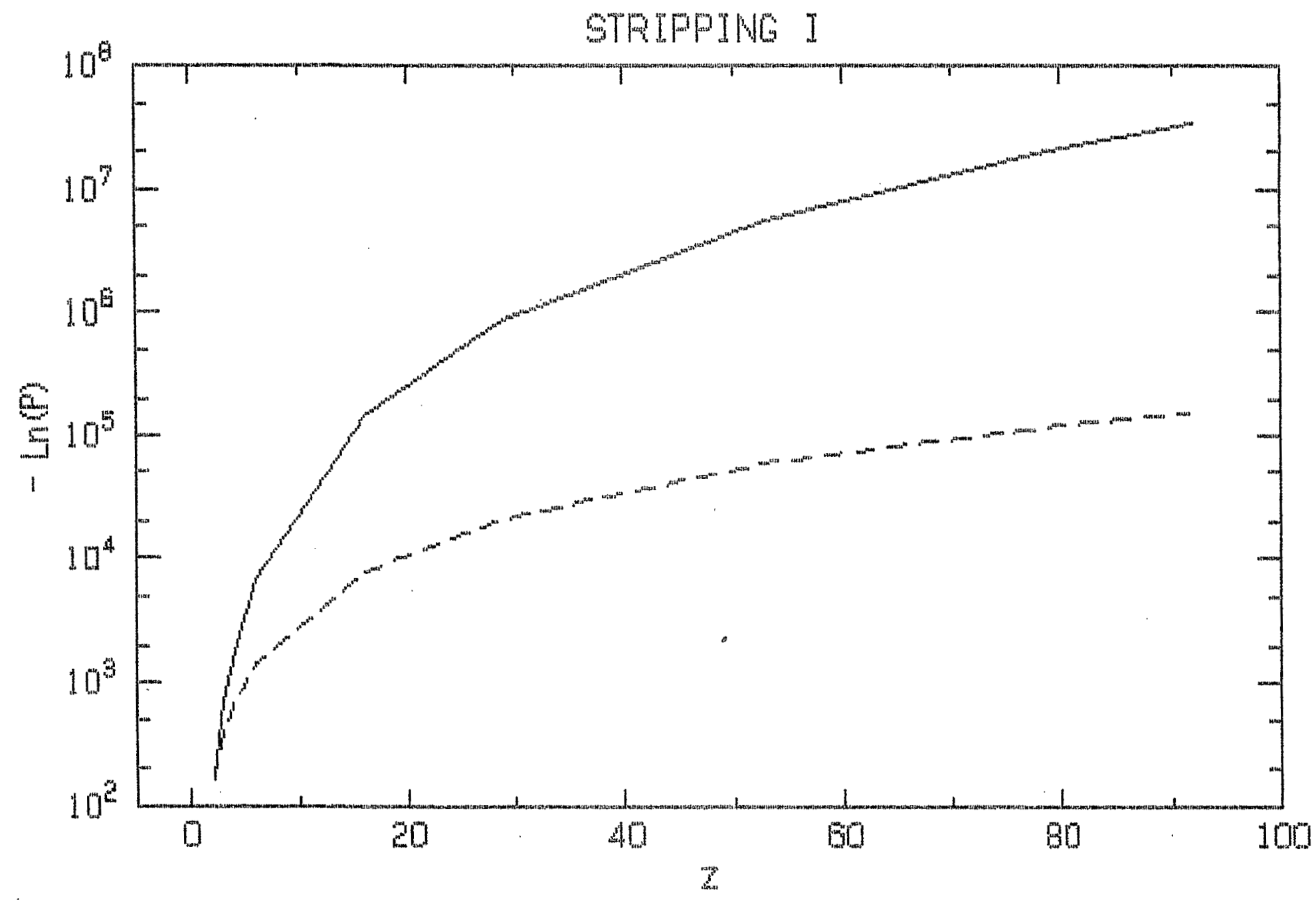

Figure 4 The logarithm of the probability versus $Z$. The solid line is from the barrier penetration method while the dashed line is from the energy density method. 


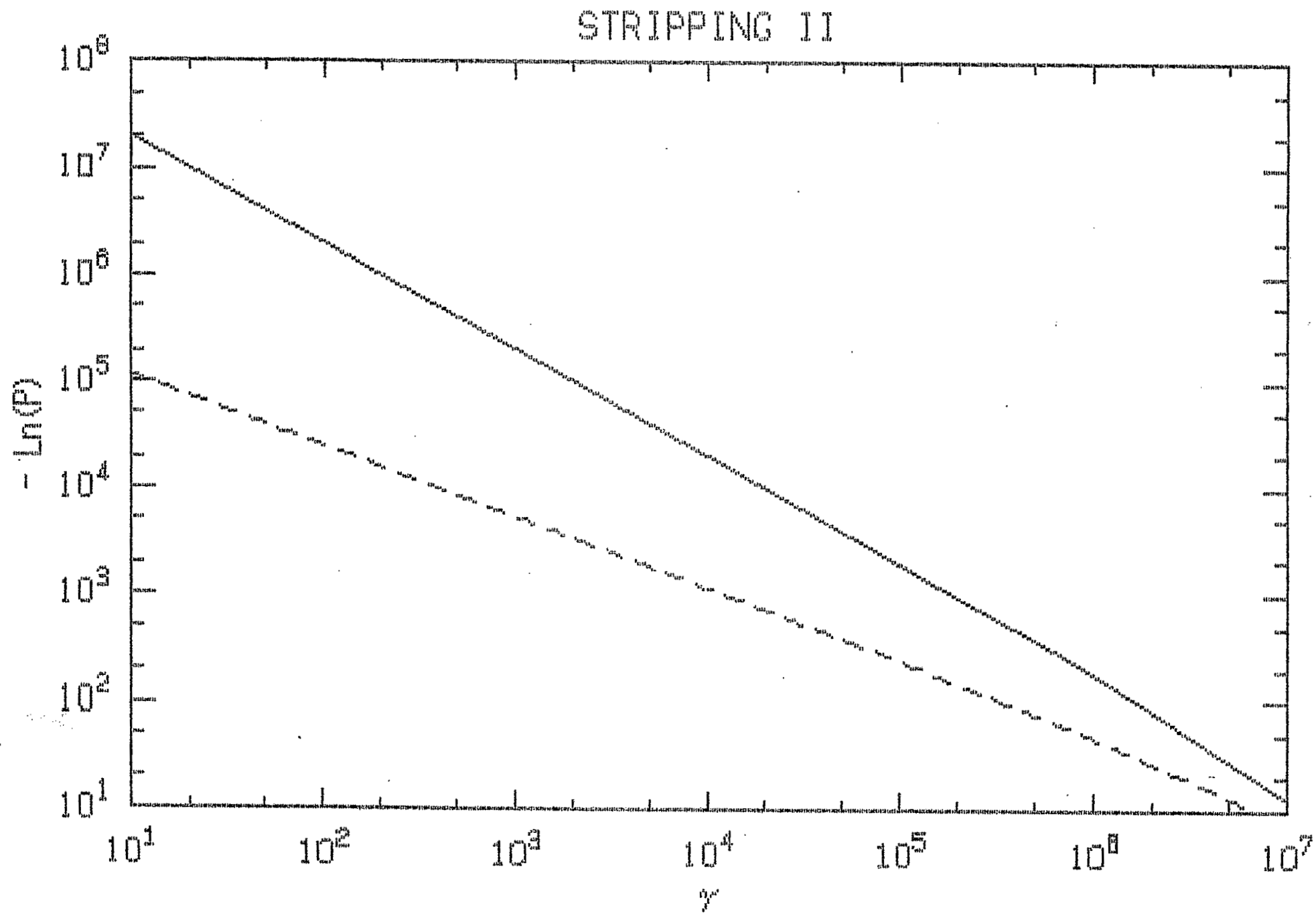

Figure 5 The logarithm of the probability versus $\gamma$. The solid line is from the barrier penetration method while the dashed line is from the energy density method. 